\title{
Impact of Time-of-Flight on Respiratory Motion Modelling using Non-Attenuation-Corrected PET
}

\author{
Alexander C. Whitehead, Student Member, IEEE, Elise C. Emond, Student Member, IEEE, \\ Nikos Efthimiou, Member IEEE, Adeyemi Akintonde, Scott W. Wollenweber, Senior Member IEEE, \\ Charles W. Stearns, Fellow, IEEE, Brian F. Hutton, Senior Member, IEEE, Jamie R. McClelland \\ and Kris Thielemans, Senior Member, IEEE
}

\begin{abstract}
Respiratory motion reduces image quality in Positron Emission Tomography (PET). Unless gated Computed Tomography (CT) or Magnetic Resonance (MR) data are available, motion correction relies on registration of the PET data. To avoid mis-registration due to attenuation mismatches, most existing methods rely on pair-wise registration of Non-Attenuation Corrected (NAC) PET volumes. This is a challenging problem due to the low contrast and high noise of these volumes. This paper investigates the possibility of using motion models for respiratory motion correction in PET, and in particular whether incorporating Time-of-Flight (TOF) information increases the accuracy of the motion models derived from the NAC reconstructed images. 4D Extended Cardiac-Torso (XCAT) phantom simulations are used for one bed position with a field of view including the base of the lungs and the diaphragm. A TOF resolution of $375 p s$ is used. NAC images are reconstructed using Orded SubSet Expectation Maximisation (OSEM) and used as input for motion model estimation. Different motion models are compared using the original XCAT input volumes. The results indicate that TOF improves the accuracy of the motion model considerably.
\end{abstract}

\section{INTRODUCTION}

$\mathbf{R}$ ESPIRATORY motion causes artefacts and loss of resolution in the thoracic region in PET [1]. Many methods have been proposed to correct for respiratory motion, usually involving registration between a reference volume and a set of volumes in different positions in the respiratory cycle obtained by gating [2]. However, such pair-wise registration is sensitive to noise. It also does not allow prediction of the respiratory

Manuscript recieved 2nd of January 2020.

Alexander C. Whitehead, Elise C. Emond, Adeyemi Akintonde, Brian F. Hutton and Kris Thielemans are with the Institute of Nuclear Medicine, University College London, London, NW1 2BU, UK (contact: alexander.whitehead.18@ucl.ac.uk).

Alexander C. Whitehead, Adeyemi Akintonde and Jamie McClelland are with the Centre for Medical Image Computing, University College London, London, NW1 2BU, UK.

Nikos Efthimiou is with the PET Research Centre, Faculty of Health Sciences, University of Hull, Hull, HU6 7RX, UK.

Scott Wollenweber and Charles Stearns are with Molecular Imaging \& Computed Tomography Engineering, GE Healthcare, USA

Elise C. Emond is supported by GlaxoSmithKline (BIDS3000030921).

Jamie R. McClelland is supported by a Cancer Research UK Centres Network Accelerator Award Grant (A21993) to the ART-NET consortium and a CRUK Multi-disciplinary grant (CRC 521).

This research is supported by GE Healthcare, the NIHR UCLH Biomedical Research Centre and the EPSRC-funded UCL Centre for Doctoral Training in Medical Imaging (EP/L016478/1).The software used was partly produced by the Computational Collaborative Project in Synergistic PET-MR Reconstruction, CCP PET-MR, UK EPSRC grant EP/M022587/1.

This work made use of computational support by $\mathrm{CoSeC}$, the Computational Science Centre for Research Communities. state for data not used to estimate the motion, for instance, to be used for real time motion correction. Surrogate driven motion models attempt to overcome these deficiencies by relating the motion in the data to a number of surrogate signals [3]. The model outputs a transformation or deformation field for every value of the surrogate signals. Motion models are calculated on a series of either time or gating based volumes.

The benefits of using attenuation correction for PET image registration are unclear. If images are reconstructed using a static Attenuation Map (mu-map), then artefacts caused by the misalignment between the activity distribution and the mumap would hamper image registration. It could therefore be advantageous to estimate motion on NAC images [4]. However, contrast may be too low to calculate an accurate motion model and artefacts associated with the mismatch between the acquisition and system model could also obscure the underlying motion.

In the absence of TOF, there is no information on the activity position along the Line-of-Responce (LOR) and NAC reconstructions have high intensity near the surface and low contrast in the internal part of the body. In TOF, the time information constrains the activity position along the LOR changing the nature and extent of the artefacts associated with NAC as well as changing noise properties [5].

The aim of this work is to investigate whether TOF can sufficiently increase the contrast and lower the noise of NAC images to facilitate the calculation of accurate motion models.

\section{MEthods}

\section{A. XCAT image generation}

XCAT [6] was used to generate 6 volumes over a linear 5 second breathing cycle, with 1 volume at full expiration at the beginning of the cycle and 1 volume at full expiration at the end of the cycle and using settings for the extent of AnteriorPosterior (AP) and Superior-Inferior (SI) motion. Activity concentrations were derived from a static Fludeoxyglucose (FDG) patient scan. The field of view included the base of the lungs, diaphragm and the top of the liver with a $40 \mathrm{~mm}$ diameter spherical lesion placed in the right lung.

\section{B. PET data simulation}

PET acquisitions were simulated using Software for Tomographic Image Reconstruction (STIR) [7], [8] through Synergistic Image Reconstruction Framework (SIRF) [9], [10] to 
forward project the input data to sinograms using the geometry of a GE Discovery 710 and, where relevant, a TOF resolution of 375ps similar to the GE Signa PET/MR (using TOF mashing to reduce computation time resulting in 13 TOF time bins of size $376.5 \mathrm{ps}$ ). Attenuation was included in the simulation using the relevant mu-map generated by XCAT. Scatter and randoms were not taken into account in the simulation. Multiple noise realisations were generated to simulate an acquisition as if it had been gated into 6 bins over an acquisition of $120 \mathrm{~s}$, emulating a standard single bed position acquisition.

\section{Image reconstruction}

Data were reconstructed without attenuation correction using OSEM with 2 full iterations and 24 subsets [11]. Volumes were post filtered using a Gaussian blurring with a kernel size of $6.4 \mathrm{~mm}$ Full Width at Half Maximum (FWHM).

\section{Motion model estimation}

3D B-splines were used to model spatial deformations with the corresponding warping operation denoted as $\mathbf{W}\left(\alpha_{t}\right)$, with $\alpha_{t}$ a vector with B-spline coefficients at time $t$. The breathing surrogate signals $\mathbf{s}$ contained 2 components, the AnteriorPosterior (AP) and Superior-Inferior (SI) motion signals used by XCAT. Following [12] a direct correspondence motion model was used where the B-spline coefficients at time $t$ are expressed as a linear combination of the 2 surrogate signals, $s_{1, t}$ and $s_{2, t}$ :

$$
\forall t \in\left[\left[1, n_{t}\right]\right], \quad \alpha_{k, t}:=R_{1, k} s_{1, t}+R_{2, k} s_{2, t}+R_{3, k}
$$

where $\alpha_{k, t}$ is the 3D B-spline coefficient for node $k$ at time point $t$, and $R_{i, k}$ are the model parameters.

A generalised framework unifying image registration and respiratory motion models, NiftyRegResp, was used to estimate the Respiratory Correspondence Model (RCM)s using Sum of Squared Differences (SSD) as an objective function [12].

\section{E. Evaluation}

We compared $3 \mathrm{RCMs}$, calculated from the PET XCAT volumes (gold standard), Non-Time-of-Flight (nonTOF) NAC reconstructions and TOF NAC reconstructions. To test the accuracy of the RCMs, the 3 models were used to warp the PET volume generated by XCAT at the mean breathing position to the position at each gate. These estimated volumes were then compared to the original XCAT input volumes. Difference volumes were obtained by subtracting the original XCAT volume $\mathbf{f}_{t}$ and warped volumes $\mathbf{W}\left(\alpha_{t}\right) \mathbf{f}_{\text {ref }}$ at the same gate. Mean Absolute Percentage Error (MAPE) were computed from these difference images.

In addition, the Centre-of-Mass (COM) of the lesion was also tracked over the 6 gates, by warping a volume only including the lesion in the reference position as above, and then computing the COM.

\section{RESUlTS}

TABLE I

COMPARISON OF THE MAPE BETWEEN THE GROUND TRUTH DATA AND THE VOLUMES ESTIMATED FROM THE XCAT BASED RCM, THE VOLUMES ESTIMATED FROM THE NAC NONTOF BASED RCM AND THE VOLUMES ESTIMATED FROM THE NAC TOF BASED RCM.

\begin{tabular}{||c|ccc||}
\hline MAPE & XCAT & nonTOF & TOF \\
\hline 1 & 1.95 & 8.35 & 4.18 \\
2 & 1.59 & 1.61 & 1.84 \\
3 & 2.06 & 9.91 & 5.23 \\
4 & 1.97 & 6.15 & 3.68 \\
5 & 1.65 & 4.45 & 2.52 \\
6 & 1.95 & 8.35 & 4.18 \\
\hline Mean & 1.86 & 6.47 & 3.60 \\
\hline
\end{tabular}

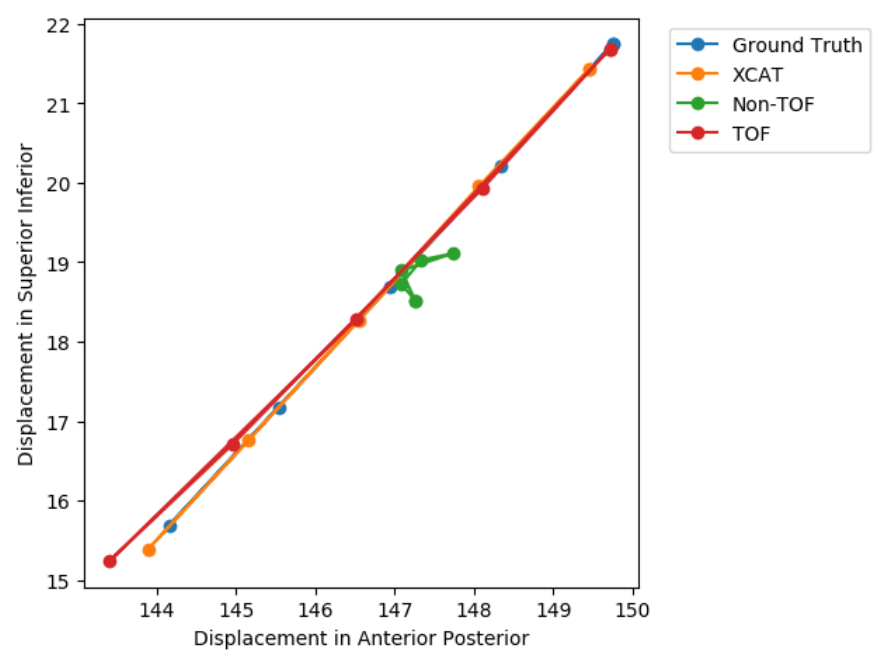

Fig. 2. The path of the COM of the lesion. Horizontal (respectively vertical) axis corresponds to motion in the AP (respectively SI) direction over the 6 gates. Different curves denote COM displacement for ground truth data, the estimated data from the XCAT based RCM, the estimated data from the NAC nonTOF based RCM and the estimated data from the NAC TOF based RCM.

The reconstructed data, estimated volumes and difference can be seen in Fig 1 and MAPE are in Table I. The mean MAPE was found to be lower for the NAC TOF data than for the NAC nonTOF.

COM results can be seen in Fig 2. The path of the NAC TOF data follows the ground truth path much closer than the NAC nonTOF data, and is quite close to the gold standard XCAT-derived motion.

\section{Discussion AND CONCLUSIONS}

Motion models derived from NAC TOF volumes were found to be more robust than when using NAC nonTOF, both visually and when comparing MAPE and COM. This was noticeable for the lung lesion in the thoracic cavity but also for other parts of the anatomy such as the liver. This is likely due to the improved image contrast of NAC TOF images. 

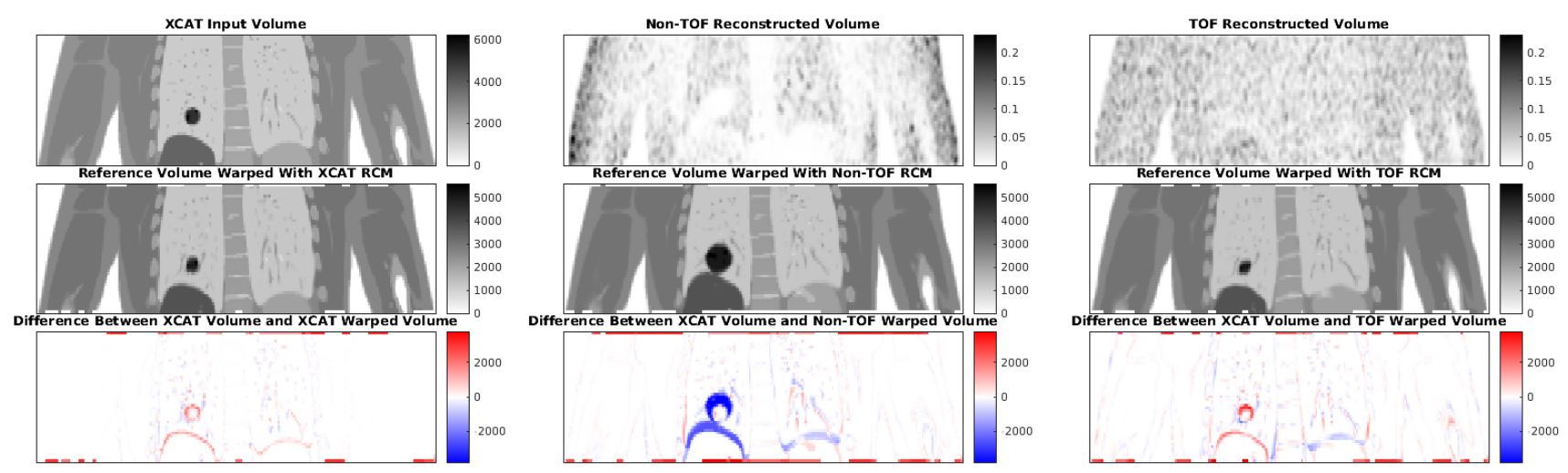

Fig. 1. All volumes correspond to end-inhalation. First row from left to right: XCAT PET data, NAC nonTOF reconstructed data and NAC TOF reconstructed data. Second row: RCM applied to mean position XCAT data with RCM derived from XCAT PET data (left), NAC nonTOF (middle) and NAC TOF (right) volumes. Colour map ranges are consistent for all images on this row. The third row from left to right: The difference between the estimated volumes from the second row with the XCAT end-inhalation volume. Colour map ranges are consistent for all images on this row.

In the future, research will focus on investigating the robustness of the motion model estimation to different noise levels, acquisition duration and size of lesion.

\section{REFERENCES}

[1] S. A. Nehmeh and Y. E. Erdi, "Respiratory Motion in Positron Emission Tomography/Computed Tomography: A Review," Seminars in Nuclear Medicine, vol. 38, no. 3, pp. 167-176, May 2008, ISSN: 0001-2998

[2] F. P. Oliveira and J. M. R. Tavares, "Medical image registration: a review," Computer Methods in Biomechanics and Biomedical Engineering, vol. 17, no. 2, pp. 73-93, Jan. 2014, ISSN: 1025-5842. DOI: 10.1080/10255842.2012.670855.

[3] J. McClelland, D. Hawkes, T. Schaeffter, and A. King, "Respiratory motion models: A review," Medical Image Analysis, vol. 17, no. 1, pp. 19-42, Jan. 2013, ISSN: 1361-8415. DOI: 10.1016/J.MEDIA.2012.09.005.

[4] Wenjia Bai and M. Brady, "Motion Correction and Attenuation Correction for Respiratory Gated PET Images," IEEE Transactions on Medical Imaging, vol. 30, no. 2, pp. 351-365, Feb. 2011, ISSN: 0278-0062. DOI: 10.1109/TMI. 2010.2078514

[5] M. M. Ter-Pogossian, N. A. Mullani, D. C. Ficke, J. Markham, and D. L. Snyder, "Photon time-of-flight-assisted positron emission tomography.," Journal of computer assisted tomography, vol. 5, no. 2, pp. 227-39, Apr. 1981, ISSN: 0363-8715.

[6] W. P. Segars, G. Sturgeon, S. Mendonca, J. Grimes, and B. M. W. Tsui, "4D XCAT phantom for multimodality imaging research," Medical Physics, vol. 37, no. 9, pp. 4902-4915, Aug. 2010, ISSN: 00942405. DOI: 10.1118/1.3480985.

[7] K. Thielemans, C. Tsoumpas, S. Mustafovic, T. Beisel, P. Aguiar, N. Dikaios, and M. W. Jacobson, "STIR: software for tomographic image reconstruction release 2," Physics in Medicine and Biology, vol. 57, no. 4, pp. 867-883, Feb. 2012, ISSN: 0031-9155.

[8] N. Efthimiou, E. Emond, P. Wadhwa, C. Cawthorne, C. Tsoumpas, and K. Thielemans, "Implementation and validation of time-of-flight PET image reconstruction module for listmode and sinogram projection data in the STIR library," Physics in Medicine and Biology, vol. 64, no. 3, p. 035004 , Jan. 2019, ISSN: 0031-9155. DOI: 10.1088/1361-6560/aaf9b9.

[9] E. Ovtchinnikov et al., "SIRF: Synergistic Image Reconstruction Framework," en, Computer Physics Communications, p. 107 087, Dec. 2019, ISSN: 0010-4655. DOI: 10.1016/j.cpc.2019.107087. [Online]. Available: http://www.sciencedirect. com/science/article/pii/S0010465519303984 (visited on 12/11/2019).

[10] E. Ovtchinnikov, R. Brown, K. Thielemans, E. Pasca, C. O. da Costa-Luis, B. Thomas, D. Atkinson, J. Mayer, A. Gillman, C. Kolbitsch, M. J. Ehrhardt, and A. Whitehead, SIRF: Synergistic Image Reconstruction Framework, version v2.1.0, Nov. 2019. DOI: 10.5281/zenodo.3548719. [Online]. Available: https://doi.org/ 10.5281/zenodo. 3548719 .

[11] H. Hudson and R. Larkin, "Accelerated image reconstruction using ordered subsets of projection data," IEEE Transactions on Medical Imaging, vol. 13, no. 4, pp. 601-609, 1994, ISSN: 02780062.

[12] J. R. McClelland, M. Modat, S. Arridge, H. Grimes, D. D’Souza, D. Thomas, D. O. Connell, D. A. Low, E. Kaza, D. J. Collins, M. O. Leach, and D. J. Hawkes, "A generalized framework unifying image registration and respiratory motion models and incorporating image reconstruction, for partial image data or full images," Physics in Medicine and Biology, vol. 62, no. 11, pp. 4273-4292, Jun. 2017, ISSN: 0031-9155. 